\title{
Inhalt des zweiundachtzigsten Bandes.
}

\author{
Erstes Heft (7. October).
}

I. Ueber das specifische Gewicht der Leber und Milz. Von Dr. H. Smidt, Assistenzarzt am stădtischen Baracken-Lazarett zu Berlin. . . . .

II. Ueber Obren - und Augenerkrankungen bei Febris recurrens. (Nach einem in dem Verein für wissenschaftliche Heilkunde zu Königsberg am 10. Mai c. gehaltenen Vortrage.) Von Dr. Luchbau, Assistenzarzt der städtischen Krankenanstalt zu Königsberg i. Pr.

III. Ueber Wanddicke und Umfang der Arterien des menschlichen Körpers. Von Valerie Schiele-Wlegandt aus Zürich. (Hlerzu 4 Tabellen.)

IV. Ueber die Folgen der Unterbindung der Ureteren und der Nierenarterien bei Thieren, im Zusammenhang mit eloigen anderen pathologischen Prozessen. Von Dr. Leo Popoff aus St. Petersburg. (Hierzu Taf. I-H.)

V. Anatomische Untersuchungen über Spondylitis. (Aus dem pathologischen Institut des Herro Prof. Langbans in Bern.) Vor Dr. Gottlieb Feurer. (Hierzu Taf. III.) .

VI. Ontersuchungen über die Entstebung von Kokken und Bakterlen in organischen Substanzen. Von Dr. Rudolf Arndt, Professor in Greifswald. (Hierzu Taf. IV.)

VII. Ueber die sogenannte Pharyngitis granulosa. Yon Dr. Martin Saalfeld in Stettin. (Aus dem pathologisch-anatomischen Institut der Universität Heidelberg.) (Hierzu Taf. V.) . . . . . . . . . . .

VIII. Ein Fall von mikroskopisch kleinen Rundwürmern - Rhabditis genitalis - im Urin einer Kranken. Mitgetheilt von Dr. S. H. S cbeiber, gew. Chef des travaux anatomiques in Bukarest, gegenw. pract. Arzt in Stuhlweissenburg (Ungarn). (Hierzu Taf. VI. Fig. 1-7.) 
IX. Woraus besteht der schwarze Punkt der Comedonen? Von Dr. P. G. Seiáte Unna in Hamburg.

X. Kleinere Mittheilungen.

1. Ueber zwei Fălle ron Anomalie der Pulmonalarterienklappen. Von Dr. A. Brüninghausen, Assistenzarzt in Pforzheim. (Hierzu Taf. VI. Fig. 8-9.)

2. Ausländiscbe Preisvertheilungen an deutsche Aerzte und Naturforscher. Zusammengestellt von Dr. W. Stricker, pract. Arzte in Frankfurt a. M. . . . . . . . . . . . . . . 2002

Berichtigung zu Bd. LXXXI. . . . . . . . . . . . . . . . . 2008

\section{Zweites Heft (5. November).}

XI. Ueber das Verbalten der kleinen Körperarterien bei Granularatrophle der Niere. Von Dr. Sotnitschewsky aus Kiew. (Aus dem pathologischen Institute des Herrn Prof. v. Becklinghausen zu Strassburg.) . . . . . . . . . . . . . . . . . . 2019

XII. Ueber angeborne Bronchiectasie. Von Dr. Paul Grawitz, Assistenten am pathologischen Institut zu Berlin. . . . . : . . . 2117

XIII. Ueber Veränderungen des Lungenepithels bei künstlich hervorgerufenen pneumonischen Prozessen. Experimentell-bistologische Untersuchungen von C. Veraguth, pract. Arzt in Zürich. (Aus dem pathologischen Institut in Zürich.) (Hierzu Taf. VII.) . . . . . . . . . .

XIV. Ein Fall von acuter Fibrinurie. Von Dr. B. Baumüller, erstem Assistenten am pathologischen Institute zu Würzburg. (Hierzu Taf. VIII.)

XV. Das Ovarialpapillom in pathologisch-anatomischer und histogenetischer Beziehung. Von Hugo Coblenz. (Pathologisches Institut zu Hallea. S.)

(Hierzu Taf. IX-XII.)..+
XVI. Ueber den Ausgang der Pnenmonie in Induration (Poeumonia fibrosa chronica. Carnificatio). Von Dr. Felix Marchand, Assistenten am pathologischen Institute zu Breslau. (Hierzu Taf. XIII.) . . . .
Neue experimentelle Beitrăge zur Inhalationstuberculose der Hunde.

XVII. Neue experimentelle Beitrăge zur Inhalationstuberculose der Hunde.
Von Dr. Tappeiner in Meran. . . . . . . . . . . .

XVIII. Bemerkungen über ein fünf Monate altes Kind mit angebornem Mangel aller Gliedmaassen. Von Dr. H. Ranke, Professor der Kinderheilkunde in Müncben. (Hierzu Taf. XIV. Fig. 3.). . . . . . . . 3660

XIX. Kleinere Mittheilungen.

Ein parasitischer Stelsszwilling. Von Dr. M. Simmonds, Assistenzarzt an der chirurgischen Klinik in Kiel. (Hierzu Taf. XIV. Fig. 1-2.) 


\section{Drittes Heft (8. December).}

XX. Belträge zur Anatomle des miliaren Tuberkels. I. Ueber LeberSeite tubereullose. Von Prof. Dr. Julius Arnold in Heidelberg. (Hierzu Taf. XV.) . . . . . . . . . . . . . . . . . .

XXI. Ueber Lupus und Tuberculose, besonders der Conjunctiva. Von Dr. Paul Bamgarten, Privatdocenten und Prosector am pathologischen Institut zu Königsberg i. Pr. (Hierzu Taf. XVI.) . . .

XXII. Ueber die Herstammung und Ausscheidung des Kalks Im gesunden und kranken Organismus. Vun Dr. Schetelig, Nervi-Homburg.

XXIII. Ueber die histologischen Veränderungen der Stauungsmilz. Von Vr. R. Nikolaides. (Hierzu Taf. XVII.) . . . . . . . . . 455

XXIV. Anatomische Notizen. (Fortsetzung.) Von Dr. Wenzel Gruber, Professor der Anatomie in St. Petersburg.

I. (CLI.) Osteologisches. . . . . . . . . . . . . . .

II. (CLII.) Schlaucbförmige, bis auf den Unterschenkel reicbende Aussackung der Synovialbaut der Capsula inter-cuneiformis 1. tarsi, vou den Vasa tibialia antica et dorsalia pedis spiralförmig unwunden. (Hierzu Taf. XVIII. Fig. 5.) . . . . . . .

III. (CLIII.) Ganglion synoviale der Capsula articularis temporomaxlllaris. . . . . . . . . . . . . . . . . . 464

IV. (CLIV.) Eine Bursa mucosa epitrochlearis subcutanea von enormer Grösse. . . . . . . . . . . . . . . .

V. (CLV.) Ueber den Musculus atlantico-basilaris, M. epistropheobasilaris und andere vor den obersten Halswirbeln zwischen den Mn. recti capitis antici majores vorkomnende Muskelvarietăten. (Hierzu Taf. XVIII. Fig. 1-4.) . . . . . . 465

VI. (CLVI.) Ein bilaminärer Musculus vastus externus biceps. . 473

VII. (CLVII.) Duplicität der Arteria occipitalis. . . . . . . 474

VIII. (CLVIII.) Verlauf der Vena anonyına sinistra vor der Tbymusdrüse. . . . . . . . . . . . . . . . .

IX. (CLIX.) Eine in die Lănge congenital vergrössert gebliebene Leber eines Erwachsenen, mit eigenthümlichem Verbalten zur Milz. .

X. (CLX.) Doppelte Nebenleber. . . . . . . . . . .

XXV. Zwei Fälle von Fibrom der Mamma mit Uebergang in Carcinom. Mitgetheilt von Dr. Max Elsaesser in Manoheim. (Aus dem pathologisch-anatomlschen Institut der Universităt Heidelberg.) (Hierzu Taf. XIX.) . . . . . . . . . . . . . . . . . . . 478

XXVI. Experimentelle Untersuchungen über die Resorption und Wirkung des regulinischen Quecksilbers der grauen Salbe. Von Dr. Paul Fürbringer, Prof. e. o. an der Universităt Jena. . . . . . 401

XXVII. Ein Beitrag zur Lungensyphilis. Von Dr. M. v. Cube, pract. Arzt in Mentone. . . . . . . . . . . . . . . . . . . 516 
XXVIII. Zwei Falle von Defect im Septum ventrlculorum nebst Verengerung der Lungenarterienbahn. Mitgetbeilt von Prof. J. O rth in Göttingen..

XXIX. Kleinere Mittheilungen.

1. Tuberculose und Perlsucht. Von Prof. E. Semmer in Dorpat.. 546

2. Die Priorităt der Entdeckung der Bakterien in der Hüboercholera, dem Milzbrand und der Rinderpest. Von Demselben.. 549

3. Die Uebertragbarkeit der Perlsucht durch die Nabrung. Von Rud. Virchow. . . . . . . . . . . . . . . . 550

4. Ueber die Folgen der Unterbindung der Ureteren und der: Nierenarterie bei Thieren in Zusammenhang mit anderen patho-logischen Prozessen. Von Dr. Johann Csokor, Adjunct und Docent des k. k. Thierarznei-Institutes in Wien.

5. Ueber eiven Sacralanhang beim Menschen. Briefliebe Mittheilung an den Herausgeber. Von Sanitätsrath Dr. de Bayter:

6. Aufforderung. Von Dr. Wilh. Stricker, pract. Arzte in Frankfurt a. M. . . . . . . . . . . . . . . . 554 